JOURNAL OF SYNCHROTRON RADIATION

ISSN 1600-5775

Received 15 September 2020

Accepted 23 March 2021

Edited by S. Svensson, Uppsala University, Sweden

Keywords: time-resolved holographic imaging; European XFEL.

Supporting information: this article has supporting information at journals.iucr.org/s

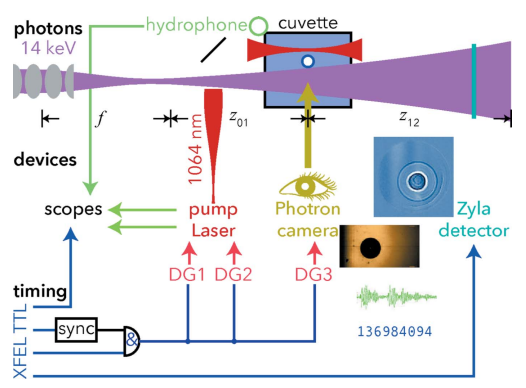

OPEN $\odot$ ACCESS

\section{Nanosecond timing and synchronization scheme for holographic pump-probe studies at the MID instrument at European XFEL}

\author{
Markus Osterhoff, ${ }^{\mathrm{a} *}$ Malte Vassholz, ${ }^{\mathrm{a}}$ Hannes Paul Hoeppe, ${ }^{\mathrm{a}}$ Juan Manuel

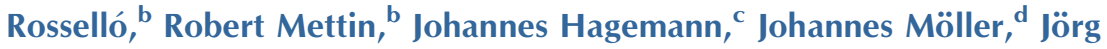 \\ Hallmann, ${ }^{\text {d }}$ Markus Scholz, ${ }^{\text {d }}$ Robert Schaffer, ${ }^{\text {d }}$ Ulrike Boesenberg, ${ }^{d}$ Chan Kim, \\ Alexey Zozulya, ${ }^{\text {d }}$ Wei Lu, ${ }^{d}$ Roman Shayduk, ${ }^{d}$ Anders Madsen ${ }^{d}$ and Tim Salditt ${ }^{a}$ \\ anstitute for X-ray Physics, University of Göttingen, Friedrich-Hund-Platz 1, 37077 Göttingen, Germany, ${ }^{\mathbf{b}}$ Third Institute \\ of Physics, University of Göttingen, Friedrich-Hund-Platz 1, 37077 Göttingen, Germany, 'Deutsches Elektronen \\ Synchrotron - DESY, Notkestrasse 85, 22607 Hamburg, Germany, and ${ }^{\mathbf{d} E u r o p e a n ~ X-r a y ~ F r e e-E l e c t r o n ~ L a s e r ~ F a c i l i t y, ~}$ \\ Holzkoppel 4, 22869 Schenefeld, Germany. *Correspondence e-mail: mosterh1@gwdg.de
}

Single-pulse holographic imaging at XFEL sources with $10^{12}$ photons delivered in pulses shorter than $100 \mathrm{fs}$ reveal new quantitative insights into fast phenomena. Here, a timing and synchronization scheme for stroboscopic imaging and quantitative analysis of fast phenomena on time scales (sub-ns) and length-scales ( $\lesssim 100 \mathrm{~nm}$ ) inaccessible by visible light is reported. A fully electronic delay-and-trigger system has been implemented at the MID station at the European XFEL, and applied to the study of emerging laser-driven cavitation bubbles in water. Synchronization and timing precision have been characterized to be better than $1 \mathrm{~ns}$.

\section{Introduction}

Strongly driven cavitation bubbles in liquids exhibit a range of interesting non-linear effects, from optical breakdown and the emission of a shockwave to violent collapse and sonoluminescence (Vogel et al., 1996a; Gaitan et al., 2010). For well controlled experiments, laser-driven cavitation events are analysed using optical and acoustical means (Vogel et al., 1996b; Johansen et al., 2017). The dynamics of laser-driven plasma, expanding and collapsing vapour bubbles have been studied by, for example, optical high-speed cameras with up to 100 million frames per second (Lindau \& Lauterborn, 2000). However, physical parameters of plasma and vapour in the early states are difficult to quantify by optical means, both due to limited temporal-spatial resolution and because optical imaging cannot directly access mass densities or gas pressures. For example, the time evolution of the bubble radius $R(t)$ close to the collapse time is yet unknown, but certainly smaller than $100 \mathrm{~nm}$.

These problems can be overcome, however, by single-pulse holography with intense X-ray light pulses (Hagemann et al., 2021). X-ray free-electron lasers (XFELs) (Altarelli et al., 2007; Saldin et al., 2000) allow an instantaneous hologram to be acquired using exposure times below $100 \mathrm{fs}$. Thanks to small detector pixel sizes below $10 \mu \mathrm{m}$ and a magnifying imaging system, a spatial resolution below $100 \mathrm{~nm}$ is achievable. Last, but not least, the reconstructed phase information directly quantifies the (projected) electron density or mass density (Gabor, 1948; Cloetens et al., 1996), even for organic matter (Giewekemeyer et al., 2011). Single-pulse holograms 


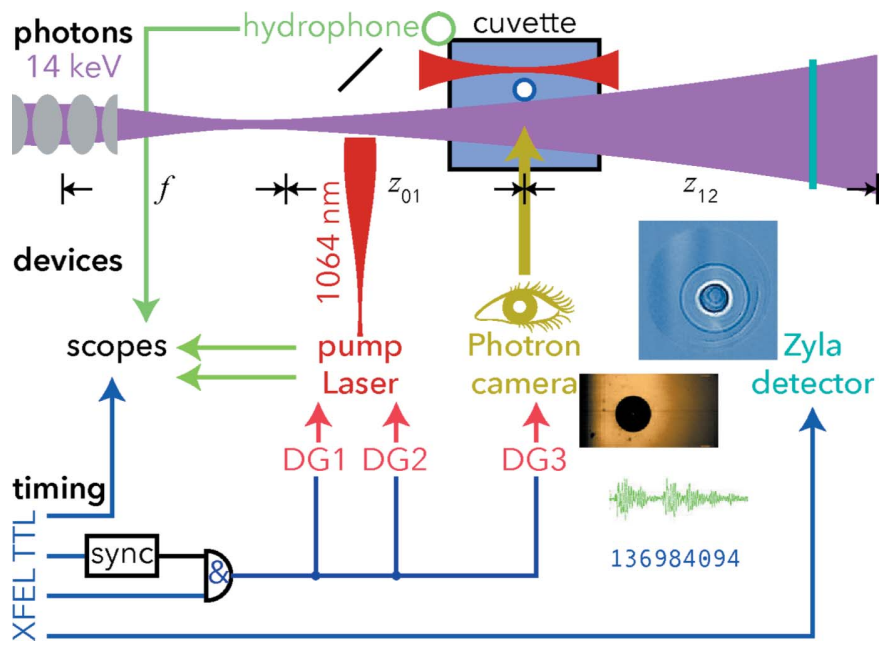

Figure 1

Overview sketch of the laser-driven cavitation X-ray imaging experiments carried out at the MID station of the European XFEL. The $1064 \mathrm{~nm}$ pump laser pulses excite cavitation events in purified water inside the cuvette. The electron density profiles of the expanding bubbles are probed by X-ray holography with well defined and adjustable time delays of a few ns up to several $\mu$ s; at lower time-resolution, optical microscopy images are recorded. Using a microphone, the bubble lifetime is measured.

offer a route to study ultra-fast processes with time-scales down to picoseconds and below. When carried out in a stroboscopic pump-probe scheme, repetitive holographic imaging delivers a time-series of the evolution of relevant physical quantities such as the radial density profile $\rho(r, \tau)$. A detailed analysis of the performed experiments is reported elsewhere (Vassholz et al., 2021).

Here we describe and analyse an electronic timing scheme with resolution better than $1 \mathrm{~ns}$ for pump-probe studies on laser-driven cavitation in water. The time-resolution is limited by the laser pulse duration of $6 \mathrm{~ns}$. Hardware to achieve synchronization and control the delay had been chosen and their compatibility with the required delay and jitter goals was tested. The total jitter is determined to be less than $0.4 \mathrm{~ns}$; this value is, however, an upper bound given by the hardware used. An overview sketch of the experiment can be found in Fig. 1.

This paper is organized as follows. First, we briefly review the European XFEL facility and the MID end-station; then we describe the performed experiments, the optical scheme, and the detectors. In Section 2.4, the delay and synchronization chain is described, and results on performance characterization are shown in Section 3. Before the paper concludes with a summary and outlook, energy fluctuations of the laser-driven cavitation bubbles are analysed.

\section{Experimental setup}

\subsection{European XFEL MID end-station}

Experiments were carried out at the Materials Imaging and Dynamics (MID) instrument of the European X-ray FreeElectron Laser (EuXFEL) in Schenefeld, Germany. The European XFEL is a hard X-ray free-electron laser developed for high-repetition-rate experiments delivering up to 27000 pulses per second (Altarelli et al., 2007).

This design value of 2700 pulses per train at a pulseseparation down to $222 \mathrm{~ns}$ and train-repetition rate of $10 \mathrm{~Hz}$ has recently been achieved. These trains repeat with a rate of $10 \mathrm{~Hz}$, with $99.4 \mathrm{~ms}$ dead-time, e.g. for detector read-out and re-charging of the experimental systems. For the experiments discussed here, however, only a single or two pulses per $10 \mathrm{~Hz}$ train were used.

In the European XFEL linear accelerator, multi-GeV electrons are directed into insertion devices (undulators) with a magnetic length of up to $175 \mathrm{~m}$, where self-amplified spontaneous emission (SASE) radiation pulses of high spatial and temporal coherence are generated (Saldin et al., 2000). In addition, the X-ray pulses are very short in time ( $\lesssim 100 \mathrm{fs}$ ), thus allowing for pump-probe experiments of very high temporal resolution. The photon flux per pulse is comparable with synchrotron radiation fluxes per second; this allows for single-pulse holographic imaging experiments to capture the time-evolution of the stroboscopically probed specimens with real-space images (Gabor, 1948; Cloetens et al., 1996). From reconstructed holograms, phase-shifting structures next to absorbing ones also become visible. Single-pulse holography thus becomes an enabling technique to study fast processes in optically thin samples, e.g. biological matter (Giewekemeyer et al., 2011).

The MID instrument is situated in the SASE-2 section of the EuXFEL and designed for experiments between $5 \mathrm{keV}$ and $25 \mathrm{keV}$ (Madsen et al., 2013, 2021). For the experiments p2207 (14 keV) and p2544 (17.8 keV), the machine was tuned for the so-far highest photon energies. The X-ray pulse energy was measured to be fluctuating around $610 \pm 260 \mu \mathrm{J}$.

The CRL-1 system is the first component of the SASE-2 beamline and can be used for pre-focusing or collimating the beam. Spot sizes below $200 \mu \mathrm{m}$ in the sample plane can be achieved. A microfocused beam can be obtained with the CRL-2 system (Madsen et al., 2013, 2021). The sample chamber can be evacuated; here, instead, experiments were carried out in air.

To achieve a large magnification for high-resolution holograms, a stack of nano-CRLs including an aberration correcting phase plate (Seiboth et al., 2017) was placed in front of the sample stage. The imaging detector was placed about $9.7 \mathrm{~m}$ downstream from the sample; the flight path between sample and detector was evacuated to avoid background from air scattering. For optical pumping of cavitation events, an infrared laser system was installed close to the sample; see below for details.

For more information refer to Table 1.

\subsection{Excitation laser, cavitation events}

To stimulate cavitation events, a nanosecond infrared laser system was used. More specifically, the $1064 \mathrm{~nm}$ emission from a Litron Lasers Nano L 200-10 (Litron Lasers, Rugby, UK) was focused into the purified water inside the cuvette. The pulse duration was about 6 ns. For beam transport, standard 
Table 1

Physical and geometrical parameters of the holographic pump-probe studies.

\begin{tabular}{lllll}
\hline & $\mathrm{p} 2207$ & & $\mathrm{p} 2544$ & \\
\cline { 2 - 2 } Parameter & May 2019 & & October 2019 & Unit \\
\hline SASE 2 & 25 & 31 & Undulator cells \\
Electron energy & 14.0 & 16.5 & $\mathrm{GeV}$ \\
Photon energy & 14.0 & 17.8 & $\mathrm{keV}$ \\
Pulse energy & $\sim 610$ & $\leq 700$ & $\mu \mathrm{J}$ \\
Repetition rate & 10 & 10 & $\mathrm{~Hz}$ (single bunch) \\
& & & \\
CRL focal length & 298 & 475 & $\mathrm{~mm}$ \\
CRL numerical aperture & 0.42 & 0.27 & $\times 10^{-3}$ \\
Distance: focus-sample & 146.5 & 276.8 & $\mathrm{~mm}$ \\
Distance: sample-detector & 8858 & 9665 & $\mathrm{~mm}$ \\
Demagnified pixel size & 97 & 181 & $\mathrm{~nm}$ \\
Fresnel number $F^{1}$ & 7.6 & 17.5 & $\times 10^{-4}$ \\
WAXS detector distances & - & $\sim 300$ & $\mathrm{~mm}$ \\
WAXS detector pixel size & - & 50 & $\mu \mathrm{m}$ \\
Laser wavelength & 1064 & 1064 & $\mathrm{~nm}$ \\
Laser pulse energy & 24 & 17 & $\mathrm{~mJ}$ \\
Laser spot size & 2 & 2 & $\mu \mathrm{m}$ (FWHM) \\
Laser pulse duration & 6 & 6 & $\mathrm{~ns}$ \\
\hline
\end{tabular}

optical components were used, including a telescope to widen the beam in front of the focusing optic. For focusing, a singlet lens was used while a numerical aperture of about 0.2 is reached in both experiments. The focused laser spot size is expected to exceed the Gaussian limit of $2 \mu \mathrm{m}$ (FWHM), since the focusing geometry is slightly affected during fine adjustment of the X-ray and laser alignment. In addition the converging beam was reflected by a drilled-through flat mirror which is expected to slightly distort the beam.

The laser intensity was set to about $21 \%-25 \%$, corresponding to about $17 \mathrm{~mJ}$ to $24 \mathrm{~mJ}$ per pulse. The seeding rate of cavitation events is about $23 \%$ with an RMS variation of $3 \%$; multi-bubble excitation is observed for $30 \%$ of the cavitation events.

Typical cavitation bubbles observed have a maximum radius of about $500-750 \mu \mathrm{m}$ and lifetimes of $100-150 \mu \mathrm{s}$; see Section 3.2 for a detailed analysis.

\subsection{X-ray focusing and detection}

2.3.1. Compound refractive lenses. For focusing of the $\mathrm{X}$-rays, a stack of 50 compound refractive lenses (CRLs; made from beryllium with an apex radius of $r=50 \mu \mathrm{m}$ and a geometrical aperture of $D=300 \mu \mathrm{m}$ ) with a focal length of $f=298 \mathrm{~mm}$ was used. The focal length is measured from the centre of the CRL stack. The CRL stack is aberration corrected by a phase-plate (Seiboth et al., 2017), thus yielding a diffraction-limited focus size of $78 \mathrm{~nm}$ (p2207) and $94 \mathrm{~nm}$ (p2544) (Hagemann et al., 2021); for further details, see Table 1.

2.3.2. X-ray detectors. For detection of holograms, a scintillator-based sCMOS camera (Andor Zyla 5.5, Oxford Instruments, Abingdon, United Kingdom) with 5 Mpixels of $6.5 \mu \mathrm{m}$ pitch was placed about $9.7 \mathrm{~m}$ downstream of the cuvette. This leads to a geometrical magnification of 62 and, accordingly, to a virtual pixel size of $106 \mathrm{~nm}$. The Fresnel
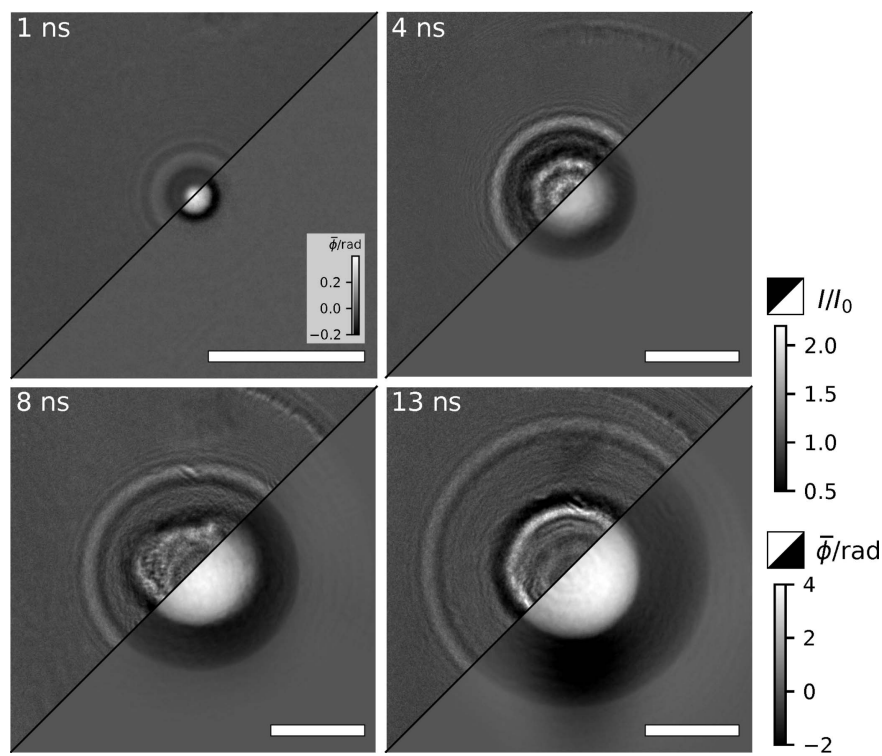

Figure 2

Flat-field corrected holograms (upper left triangles) and reconstructed phase (lower right triangles) of four cavitation events at increasing time delay. The scale bars denote $25 \mu \mathrm{m}$.

number of the wave-optical imaging system is about $F \simeq$ $7.6 \times 10^{-4}$. See Table 1 for a summary of these and additional parameters for both experiments. The given corrected Fresnel number is based on visual inspection of different reconstructions and includes corrections for, for example, a curvature of the incoming wavefront by upstream optics, uncertainties in the measured distances, and possible distortions by the visual light optics of the X-ray detector.

Exemplary flat-field corrected holograms and phase reconstructions of four cavitation events with increasing time delay are shown in Fig. 2. For further details, the reader is referred to Hagemann et al. (2021).

2.3.3. Optical camera and microphone. In addition to the X-ray detector, a high-speed optical camera (Photron Fastcam SA5, up to 50000 frames per second at $512 \times 272$ pixels; Photron, Tokyo, Japan) was also used to obtain image series of the bubble growth and collapse processes. Based on the images, the maximum bubble radius can be measured to estimate the deposited energy per individual cavitation event.

As an event marker, a piezo-electric microphone was attached to the water cuvette, serving as a microphone and recording acoustics generated by the plasma ignition and the bubble collapse. From these, the bubble lifetime $\tau \simeq$ $\mathcal{O}(100 \mu \mathrm{s})$ can be extracted. Correlating $\tau$ to the maximum bubble radius obtained from the Photron camera images allows estimating the deposited energy, and distinguishing differently seeded bubbles. The reader is referred to Section 3 below and Vassholz et al. (2021) for more details.

\subsection{Time delays and triggering}

The timing scheme with all trigger signals, the laser pump pulses, the X-ray probe flashes, and detector image data including phase reconstructions, is available as an animated 


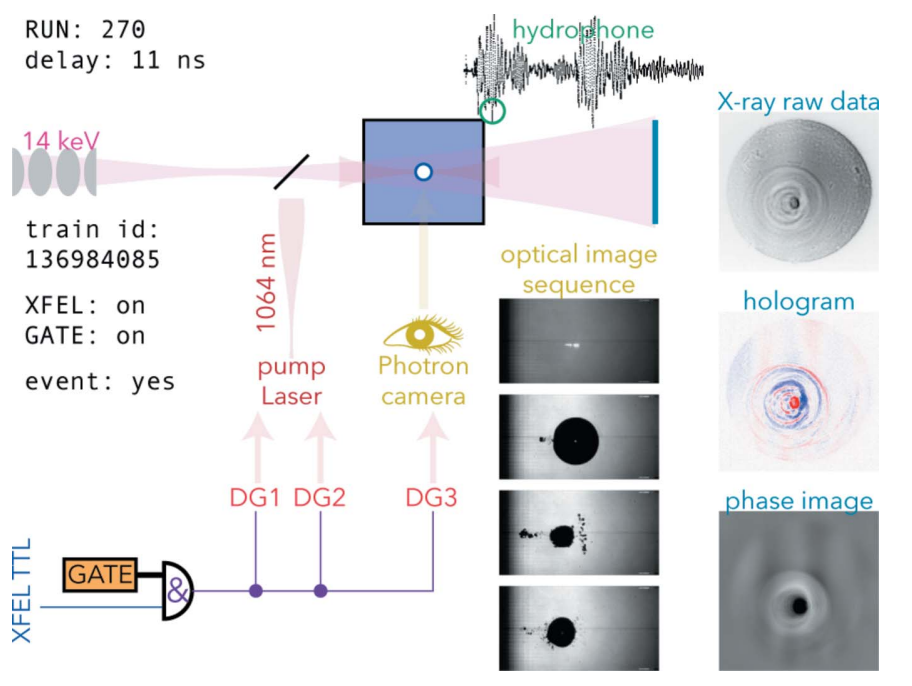

Figure 3

One particular cavitation event (the full animation is available as an animated movie in the supporting information), shown as optical microscopy (central column of grey-scale images) and X-ray detector images (top image, right), including normalized hologram (blue-red coloured image) and phase image (grey scale, bottom image, right). The cavitation event was detected using the microphone signal (oscilloscope trace, top).

movie in the supporting information. One still image is shown in Fig. 3; here, the AND gate is switched on, an actual cavitation event was pumped and recognized by the microphone, and corresponding data taken with optical camera and X-ray detector are shown. Also, the normalized hologram and the reconstructed phase image are shown.

2.4.1. Cabling scheme. With the timing specifications of the European XFEL facility in mind, the time-resolution of $<1 \mathrm{~ns}$ in this work is very modest; also the requirements on cables and connections are rather low. Hence, usual co-axial RG 58 $\mathrm{C} / \mathrm{U}$ cables with $50 \Omega$ impedance and BNC connections have been used. At a length of $20 \mathrm{~m}$, they are rated for a bandwidth of $\sim 340 \mathrm{MHz}$ (at $3 \mathrm{~dB}$ amplitude attenuation); this allows for nearly unaffected delivery of the signals to oscilloscopes. Inside the control hutch, cables are much shorter and the bandwidth is of virtually no concern.

The full cabling scheme is sketched in Fig. 4. Blue lines carry the initial EuXFEL trigger signals (see also the top part of Table 2), continuously at $10 \mathrm{~Hz}$. The purple lines show the gated signals, which are only active during measurement. Lines shown in red are electronically delayed to define the current time delay. The green lines are used to measure and quantify the timing using the PicoScope; lines depicted in orange carry debugging signals to the oscilloscope for visual inspection (see below for details on the oscilloscopes).

2.4.2. Fan-out and AND gate. To synchronize all equipment, the same first pulse has to be propagated deterministically to all devices. To this end, a PoKeys57E board was used to 'measure' the $10 \mathrm{~Hz}$ period of the EuXFEL, and an AND gate (74ACT08 with AD8009 amplifier) was introduced before a 1:4 TTL fan-out (PRL-414B, Pulse Research Lab, Torrance, California, USA). For the fan-out, the bandwidth of $>80 \mathrm{MHz}$, a (typical) rise time of $2.2 \mathrm{~ns}$ and a channel skew of $0.5 \mathrm{~ns}$, the
Table 2

Triggers from EuXFEL and (delayed) triggers to the experiment.

\begin{tabular}{|c|c|c|}
\hline Name & Scope & Purpose \\
\hline XFEL trigger 1 & A & $T_{0}$ reference trigger for oscilloscopes \\
\hline XFEL trigger 2 & & Input to delay chain (below) \\
\hline XFEL trigger 3 & & Trigger for AND gate synchronization \\
\hline XFEL trigger 4 & & Trigger for X-ray detector (Zyla) \\
\hline \multicolumn{3}{|l|}{ AND gate, then } \\
\hline fan-out-0 & D0 & Gated trigger pulses \\
\hline fan-out-1 & & To DG1 \\
\hline fan-out-2 & & To DG2 \\
\hline fan-out-3 & & To DG3 \\
\hline \multirow[t]{2}{*}{ DG1 } & D1 & Input laser flash \\
\hline & D2 & Output laser flash \\
\hline \multirow[t]{2}{*}{ DG2 } & D3 & Input laser Pockels \\
\hline & B & Output laser Pockels \\
\hline \multirow[t]{4}{*}{ DG3 } & D4 & Input Photron camera \\
\hline & $\mathrm{C}$ & Output Photron camera \\
\hline & $\mathrm{D}$ & Output microphone \\
\hline & D5 & Output X-ray detector (Zyla) \\
\hline
\end{tabular}

timing requirements of the experiment are easily met. For the AND gate and amplifier, a propagation delay of $3.55 \mathrm{~ns}$ and a rising time of $2.3 \mathrm{~ns}$ was measured. The combined delay of fan-out and AND gate was measured to be $19.0 \mathrm{~ns}$ with RMS $0.1 \mathrm{~ns}$.

2.4.3. Delay generators. A pair of low jitter delay generators DG535 (Stanford Research Systems) was utilized to define precisely timed pulses for the laser flash lamp ( $\left.T_{\text {flash }}\right)$, the Pockels cells ( $T_{\text {Pockels }}$ ), and the high-speed optical camera ( $\left.T_{\text {photron }}\right)$. The delay between $T_{\text {flash }}$ and $T_{\text {Pockels }}$ was kept constant at $160 \mu$ s to maximize the lasing power. Then, by shifting $T_{\text {flash }}$ with respect to EuXFEL's trigger $T_{0}$, the delay between pumping laser pulse and probing X-ray pulse was varied from negative values (first X-ray, then laser) to positive values, with a dense sampling close to $0 \mathrm{~ns}$ and a coarse sampling up to $11 \mu \mathrm{s}$. This is possible since the actual EuXFEL photon train arrives after the electronic trigger signal.

The time delay XFEL trigger 4 of the X-ray detector was preset to the value 5212395 FPGA clock cycles; the exposure

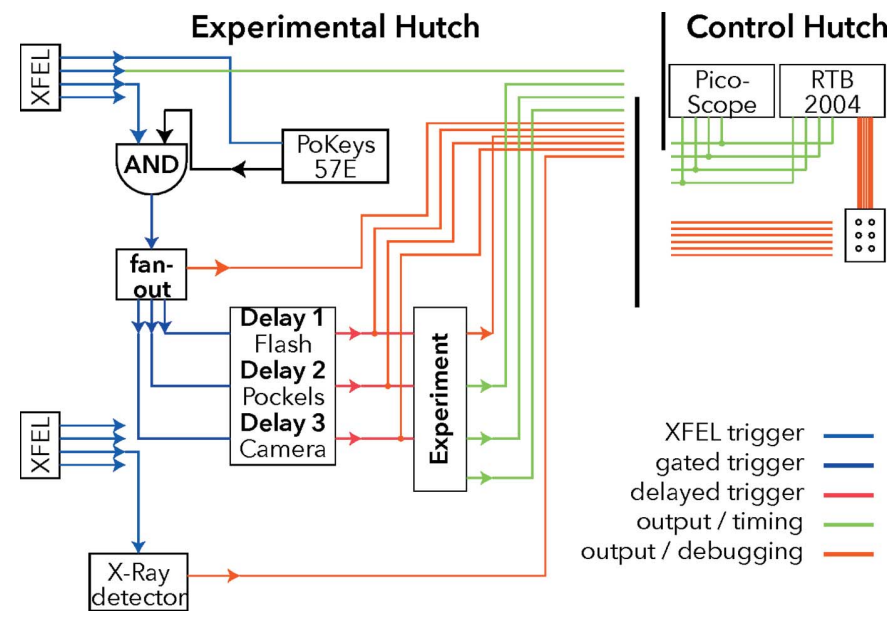

Figure 4

Schematics of the BNC cabling; for details see the text. 
time was set to half the EuXFEL period, i.e. $50 \mathrm{~ms}$. Since the EuXFEL pulses have a duration on the order of $100 \mathrm{fs}$ and repeat with $10 \mathrm{~Hz}$, the actual exposure length and phase are non-critical for the experiment. It has to be noted, though, that the event stamping with respect to the trainId needs to be checked. In the presented experiments, the X-ray detector was lagging behind by one trainId, but this has been taken care of in the data analysis.

2.4.4. Oscilloscopes. All timing signals were visually inspected using a stand-alone oscilloscope RTB 2004 (Rohde \& Schwarz, Munich, Germany; four analog channels plus six out of 16 digital on-off-channels with a bandwidth of $300 \mathrm{MHz}$; sampling rate up to $2.5 \mathrm{GS} \mathrm{s}^{-1}$ ); for data processing, the four analog channels were also digitized by a USB oscilloscope (PicoScope 6402C, Pico Technology, St Neots, UK: four channels, bandwidth $250 \mathrm{MHz}$, per-channel sampling rate up to $1.25 \mathrm{GS} \mathrm{s}^{-1}$ ). The four analog channels are:

A - EuXFEL trigger pulse ( $T_{0}$, XFEL trigger 1$)$;

$\mathrm{B}$ - Laser Pockels out $\left(T_{\text {Pockels }}\right)$;

C - Photron out ( $\left.T_{\text {photron }}\right)$;

D - Microphone signal (event marker).

On channel D, residual noise signals emerging from the Pockels cells can also be seen.

The six digital channels are mainly used for debugging/ visual inspection of input signals or uncritical signals (i.e. laser flash in/output and X-ray detector output).

Apart from timing and event marking, the microphone signal is also used to quantify the deposited energy, and, derived from that, the maximum bubble size. The data have therefore been calibrated to Photron images; the camera was not used during all runs because of its slow data transfer.

2.4.5. Triggers from EuXFEL. The trigger signal XFEL trigger $1\left(T_{0}\right)$ from the EuXFEL was recorded along with output signals from laser Pockels ( $\left.T_{\text {Pockels }}\right)$ and the Photron camera $\left(T_{\text {photron }}\right)$ and the microphone to measure per-shot delay values of the experiment. A signal before the AND gate and fan-out had been chosen as a reference to catch jitter of this equipment as well. For the synchronization, the signal XFEL trigger 3 had been monitored before the start of a run in order to switch the AND gate at a definite phase. Then signals from XFEL trigger 2 could pass the AND gate and fanout, to be propagated to three delay generator channels for the actual experiment. See Table 2 for more details, Fig. 4 for a schematic of the cabling, and Fig. 5 for exemplary oscilloscope traces.

The EuXFEL trigger signals are generated by local FPGA boards, which are interconnected via glass fibre to the master oscillator at DESY. They allow for per-output shifts in clocks of $9.23 \mathrm{~ns}$.

2.4.6. Spatial and temporal overlap. To find the temporal overlap, a photodiode AXUVHS11 (Opto Diode, Camarillo, California, USA; rise time $700 \mathrm{ps}$ ) was placed into the fully diverged laser and EuXFEL beam at $\sim 8 \mathrm{~m}$ distance from the interaction region. Using the DG535 delay generators, the pump-laser was shifted to temporally overlap with the EuXFEL flashes. The correct timing was later confirmed during the pump-probe experiments.

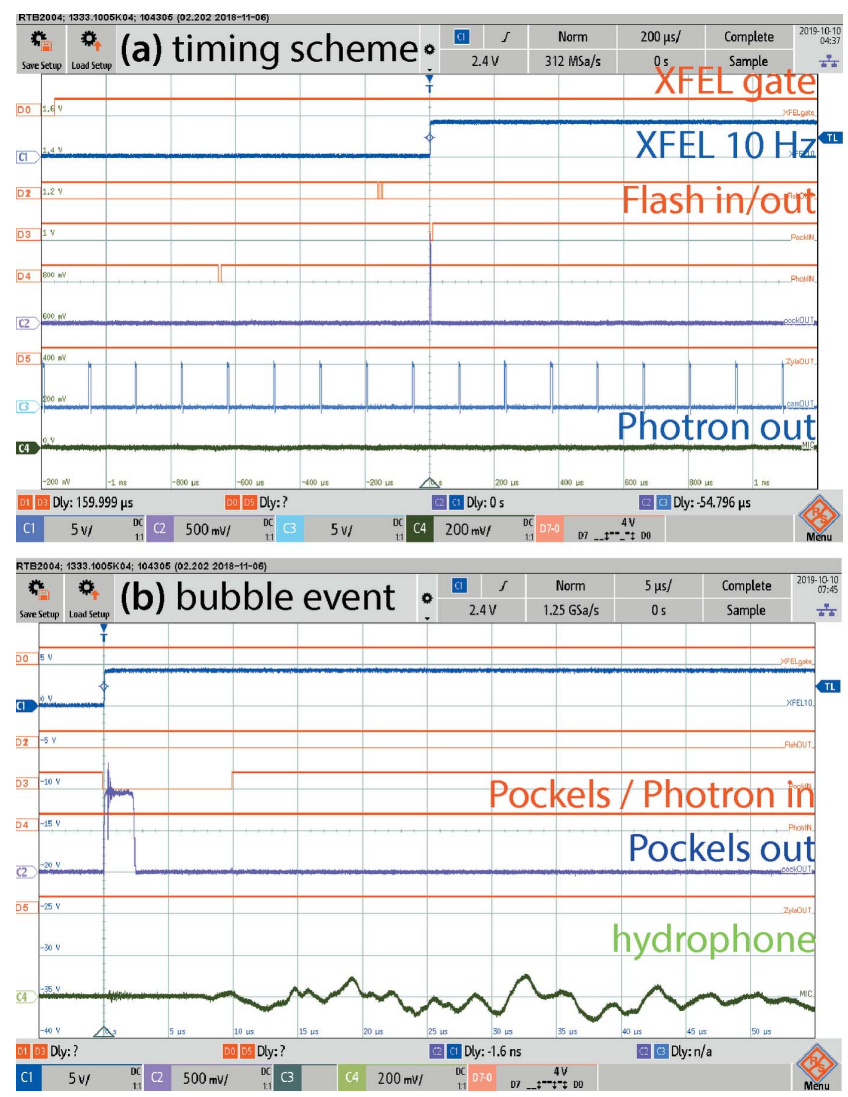

Figure 5

Oscilloscope traces: analog channels are shown in individual colours, digital channels in orange. (a) The overall timing scheme shows the master EuXFEL trigger (second trace) and the trigger pulses to/from laser (flash and Pockels), optical Photron and X-ray Andor Zyla camera. (b) A bubble event is registered by the microphone (last trace). Residual noise from the Pockels cells (sixth trace) has been used to measure actual time delays and jitter (see also Fig. 6).

To spatially align the pumping laser beam and probing EuXFEL beam, a motorized tungsten (W) needle was placed close to the interaction region and then aligned in X-ray absorption contrast. Then the optical laser was aligned onto the needle using motorized mirrors. For the liquid jet experiments, the thin water jet (diameter around $30 \mu \mathrm{m}$ ) was aligned with respect to the needle, too. The correct spatial alignment was confirmed and corrected during the actual pump-probe experiments.

\section{Characterization and control}

\subsection{Jitter and delay measurements}

The jitter of the synchronization chain (AND gate, fan-out) and laser have been determined from characteristic noise induced by the Pockels cells into a BNC cable. The PicoScope was triggered by the EuXFEL $T_{0}$, and the time delay to the Pockels signal has been quantified for a particular delay campaign. The PicoScope was operated at a sampling interval of $0.8 \mathrm{~ns}$ and with an analog bandwidth of $250 \mathrm{MHz}$; the estimated jitter of $0.4 \mathrm{~ns}$ is thus an upper bound. The nominal 
and measured delay values are in agreement within a margin of $0.2 \mathrm{~ns}$ for analog channels and within $0.5 \mathrm{~ns}$ for digital channels.

Also, delays and jitter introduced by the timing equipment and cables have been measured using the RTB scope at a sampling rate of $0.8 \mathrm{~ns}$ down to $0.4 \mathrm{~ns}$ (depending on settings; the analog bandwidth is $300 \mathrm{MHz}$ ). The channel skew of the TTL fan-out including two long BNC cables was measured to be $0.6 \mathrm{~ns}$. BNC extensions to reach the optical high-speed Photron camera (+24 ns) and the microphone at the cuvette (+26 ns) have been measured, as well as the ' 0 '-delay of the DG535 delay generators ( $88.4 \mathrm{~ns}$ to $90.5 \mathrm{~ns}$, depending on channel and device). In addition to the four analog channels, the RTB offers a logic probe with up to 16 channels; the propagation delay (probe plus ribbon cable) was determined to be $7.7 \mathrm{~ns}$. From the measurements, all delay values given in this paragraph can be considered to be 'constant' within $0.2 \mathrm{~ns}$ (standard deviation of the RTB's delay measurements).

Apart from these individual and ex situ measurements, the full delay jitter can also be quantified. For that we make use of the strong noise-like, but reproducible, pattern obtained in long BNC cables close to a laser operated with Pockels cells. Fig. 6(a) shows a reference trace of the signal. This has been correlated in the time-domain to measurements at different nominal delay values to directly measure the actual delay and its jitter. Histograms of representative delays are shown in Fig. 6(b), with a bin width of 0.8 ns corresponding the PicoScope's sampling rate. The calculated mean values are within $\pm 0.3 \mathrm{~ns}$ of the nominal values; the histogram distributions show a width of $\sim 0.4$ ns. We conclude that the jitter between the primary EuXFEL trigger $T_{0}$ and the actual $T_{\text {Pockels }}$ is below the time resolution goal of $1 \mathrm{~ns}$. The PicoScope was triggering on the EuXFEL reference before the AND gate, so this

(a) reference noise of Pockels cell

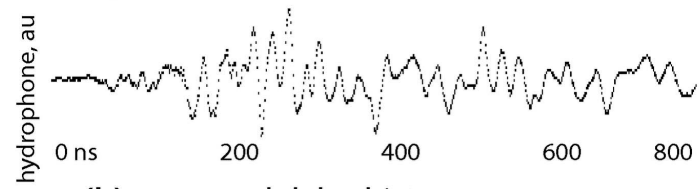

(b) measured delay histogram

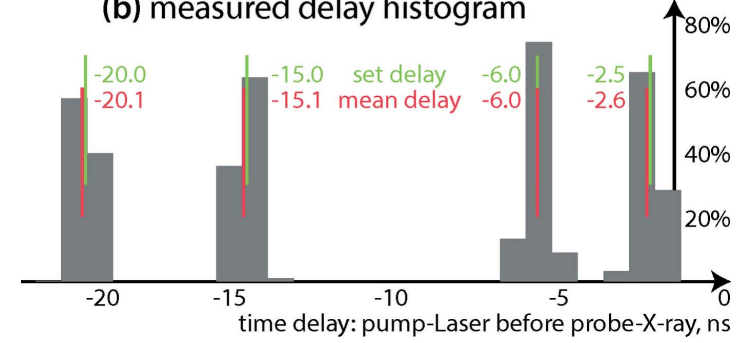

Figure 6

Pockels cell jitter measurement via noise correlation. (a) The Pockels effect induces a strong noise-like, but reproducible, pattern in a long BNC cable observed with the oscilloscopes. The reference signal shown here is correlated in the time-domain to individual signals from the time delay runs. The scope was triggered using the primary EuXFEL trigger $T_{0}$. (b) Corresponding delay histograms (grey bars) for certain nominal time delays (green lines) and average delays from cross-correlations (red lines). All measurements agree within the sampling interval of $0.8 \mathrm{~ns}$. It can thus be concluded that the jitter between primary EuXFEL trigger $T_{0}$ and the actual $T_{\text {Pockels }}$ is $\sigma \simeq 0.4 \mathrm{~ns}$. measurement includes the full delay chain comprising AND gate, fan-out, DG535, and the laser itself.

\subsection{Energy fluctuation of bubble seeding}

Holographic images and reconstructed phases of single cavitation events at a single time delay are pictorial; to study the time evolution, a series of images along a certain time delay range is needed. But the quantitative analysis of such stroboscopic experiments relies on the assumption of repetitive events. For laser seeded bubbles, the time point is well known (see analysis above) and the pulse energy can be estimated to be rather constant. But the deposited energy fluctuates per shot; hence cavitation bubbles of different sizes and hence time evolutions are produced within a single time delay run.

Such a family of bubbles can be described by a single parameter, i.e. the deposited energy E (Vogel et al., 1996a). The energy is not measured directly, but quantified from the recorded maximum radius $R_{\max }$, and with the microphone data the bubble lifetime $\tau$ (time duration between plasma ignition and collapse sound) is available. From model calculations and measurements it is known that $E, R_{\max }$, and $\tau$ are bijective functions of one another (at least within reasonable limits). Although the actual $E\left(R_{\min }\right)$ is unknown here, we can distinguish and sort different bubbles by their lifetime. Maximum radius $R_{\max }$ and bubble lifetime can be related via

$$
\tau \simeq 2 \times 0.915 R_{\max }(\rho / p)^{1 / 2},
$$

with density $\rho$ and pressure $p$, according to the rather simple Rayleigh model (Rayleigh, 1917). Note that we assume a symmetry between growth and collapse, which yields a factor of 2. While more advanced models include, for example, local pressure variations, the linear relations hold (Plesset \& Prosperetti, 1977). Fig. 7(a) shows a scatter plot of maximum radius $R_{\max }$ (taken from the Photron camera image series) and the lifetime $\tau$ (extracted from the microphone traces). Since only a part of the data streams include Photron images due to slow read-out, we have used the linear fit model,

$$
\begin{aligned}
R_{\min }(\tau) & =m \times \tau+b \\
& \simeq 4.45(3) \mathrm{m} \mathrm{s}^{-1} \times \tau+84(3) \mu \mathrm{m},
\end{aligned}
$$

to extrapolate and tag the events with an 'energy' label. The offset $R_{0} \simeq 84 \mu \mathrm{m}$ can only in part (about $30 \mu \mathrm{m}$ to $80 \mu \mathrm{m}$, depending on threshold values) be attributed to the initial plasma size of the cavitation seeding event. Figs. 7(b) and 7(c) show histograms of the bubbles' maximum radius and their lifetime, respectively.

\section{Summary and outlook}

Single-pulse holographic X-ray imaging has become a reality thanks to the extreme brilliance of XFEL sources. We have designed and analysed an electronic delay scheme for timeresolved pump-probe experiments at a time-resolution of better than $1 \mathrm{~ns}$. The delay chain is fully electronic, providing more flexible and easy manipulation during the experiment, 
(a) Bubble radius vs. lifetime

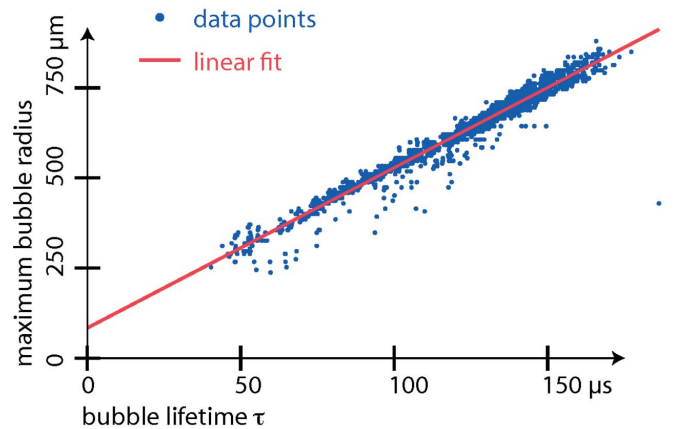

$\begin{array}{lll}\text { (b) Histogram of radius } & \text { (c) Histogram of lifetime }\end{array}$
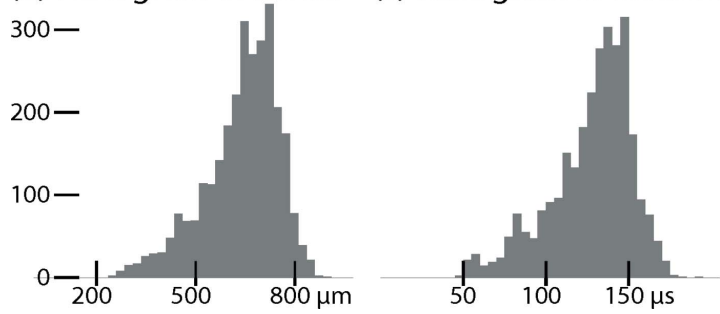

Figure 7

Energy fluctuations of cavitation events: ( $a$ ) maximum bubble radius $R_{\max }$ as a function of bubble lifetime $\tau$. $(b, c)$ Histograms of the bubbles' maximum radius and lifetime distributions, respectively.

compared with optical delay lines. The overall jitter between incoming EuXFEL trigger pulse and secondary electronic signal generated by the laser's Pockels cell has been measured in situ and quantified to be better than $0.4 \mathrm{~ns}$ (RMS); this estimate is an upper bound due to the oscilloscope's limited sampling rate.

As an event marker, a microphone signal has been recorded per X-ray pulse; these data are used both to find cavitation events and to quantify the deposited energy from the bubble lifetime. The lifetime correlates well to the bubbles' maximum radii, which are another observable related to energy. The deposited energy is needed in the analysis to group cavitation events into 'energy bins'.

For moderate time resolution using, for example, ns-laser systems, the presented scheme is sufficient. A fs-laser system equipped with optical delay lines is installed and commissioned at the European XFEL to access even shorter time scales.

The algorithm for single-pulse phase reconstruction of holograms with fluctuating intensity profiles from pulse to pulse can be found in Hagemann et al. (2021). Data taken at European XFEL are available - Hagemann et al. $(2019 a, b)$.

\section{Acknowledgements}

We acknowledge European XFEL in Schenefeld, Germany, for provision of X-ray free-electron laser beam time at Scientific Instrument MID (Materials Imaging and Dynamics) and would like to thank the instrument group and facility staff for their assistance. We are indebted to Peter Luley for excellent engineering support and thank Bastian Hartmann and Peter Nieschalk for additional mechanical work, and Jan
Goeman for help with IT questions, in particular for taking care of data storage and analysis systems. We thank the electronical and mechanical workshops in Göttingen for their support. We acknowledge Jan Stehlmann-Polewicz and Ralph Döhrmann for support. Open access funding enabled and organized by Projekt DEAL.

\section{Funding information}

Funding for this research was provided by: BMBF grant No. 05K19MG2 (NeuroTomo) and grant No. 05K16MGA (Nanosolar) BMBF 05K13OD2 (Erzeugung und Charakterisierung von nanofokussierten XFEL-Pulsen zur Abbildung ultraschneller Prozesse in Materie). Deutsche Forschungsgemeinschaft through SFB 755 'Nanoscale Photonic Imaging', projects $\mathrm{C} 01, \mathrm{C} 10$, and $\mathrm{C} 12$. MV is part of the Max Planck School of Photonics supported by BMBF, Max Planck Society, and Fraunhofer Society.

\section{References}

Altarelli, M., Brinkmann, R., Chergui, M., Decking, W., Dobson, B., Düsterer, S., Grübel, G., Graeff, W., Graafsma, H., Hajdu, J., Marangos, J., Pflüger, J., Redlin, Riley, D., Robinson, I., Rossbach, J., Schwarz, A., Tiedtke, K., Tschentscher, T., Vartaniants, I., Wabnitz, H., Weise, H., Wichmann, R., Witte, K., Wolf, A., Wulff, M. \& Yurkov, M. (2007). The European X-ray Free-Electron Laser: Technical Design Report. European XFEL Project Team, Hamburg, Germany.

Cloetens, P., Barrett, R., Baruchel, J., Guigay, J.-P. \& Schlenker, M. (1996). J. Phys. D Appl. Phys. 29, 133-146.

Gabor, D. (1948). Nature, 161, 777-778.

Gaitan, D., Tessien, R., Hiller, R., Gutierrez, J., Scott, C., Tardif, H., Callahan, B., Matula, T. J., Crum, L. A., Holt, R. G., Church, C. C. \& Raymond, J. L. (2010). J. Acoust. Soc. Am. 127, 34563465 .

Giewekemeyer, K., Krüger, S. P., Kalbfleisch, S., Bartels, M., Beta, C. \& Salditt, T. (2011). Phys. Rev. A, 83, 023804.

Hagemann, J., Möller, J. \& Salditt, T. (2019a). Cavitation dynamics studied by time-resolved high-resolution $x$-ray holography (p2207). doi:10.22003/XFEL.EU-DATA-002207-00.

Hagemann, J., Scholz, M. \& Salditt, T. (2019b). Cavitation dynamics studied by time-resolved high-resolution X-ray holography ( $\mathrm{p} 2544$, derived from p2207), doi:10.22003/XFEL.EU-DATA-002544-00.

Hagemann, J., Vassholz, M., Hoeppe, H., Osterhoff, M., Rosselló, J. M., Mettin, R., Seiboth, F., Schropp, A., Möller, J., Hallmann, J., Kim, C., Scholz, M., Boesenberg, U., Schaffer, R., Zozulya, A., Lu, W., Shayduk, R., Madsen, A., Schroer, C. G. \& Salditt, T. (2021). J. Synchrotron Rad. 28, 52-63.

Johansen, K., Song, J. H., Johnston, K. \& Prentice, P. (2017). Ultrasonics, 73, 144-153.

Lindau, O. \& Lauterborn, W. (2000). AIP Conf. Proc. 524. 385388.

Madsen, A., Hallmann, J., Roth, T. \& Ansaldi, G. (2013). Scientific Instrument Materials Imaging and Dynamics (MID). Technical Design Report DESY-2013-00872. XFEL.EU TR-2013-005, pp. 1191. European X-ray Free-Electron Laser Facility GmbH, Germany.

Madsen, A., Hallmann, J., Ansaldi, G., Roth, T., Lu, W., Kim, C., Boesenberg, U., Zozulya, A., Möller, J., Shayduk, R., Scholz, M., Bartmann, A., Schmidt, A., Lobato, I., Sukharnikov, K., Reiser, M., Kazarian, K. \& Petrov, I. (2021). J. Synchrotron Rad. 28, 637-649. Plesset, M. S. \& Prosperetti, A. (1977). Annu. Rev. Fluid Mech. 9, 145-185. 


\section{beamlines}

Rayleigh, L. (1917). London, Edinb. Dubl. Philos. Mag. J. Sci. 34, 9498.

Saldin, E. L., Schneidmiller, E. \& Yurkov, M. V. (2000). The Physics of Free Electron Lasers. Springer Science \& Business Media.

Seiboth, F., Schropp, A., Scholz, M., Wittwer, F., Rödel, C., Wünsche, M., Ullsperger, T., Nolte, S., Rahomäki, J., Parfeniukas, K., Giakoumidis, S., Vogt, U., Wagner, U., Rau, C., Boesenberg, U., Garrevoet, J., Falkenberg, G., Galtier, E. C., Ja Lee, H., Nagler, B. \& Schroer, C. G. (2017). Nat. Commun. 8, 14623.
Vassholz, M., Hoeppe, H. P., Hagemann, J., Rosselló, J. M., Osterhoff, M., Mettin, R., Kurz, T., Schropp, A., Seiboth, F., Schroer, C. G., Scholz, M., Möller, J., Hallmann, J., Boesenberg, U., Kim, C., Zozulya, A., Lu, W., Shayduk, R., Schaffer, R., Madsen, A. \& Salditt, T. (2021). Nat. Commun. Accepted.

Vogel, A., Busch, S. \& Parlitz, U. (1996a). J. Acoust. Soc. Am. 100, 148-165.

Vogel, A., Nahen, K., Theisen, D. \& Noack, J. (1996b). IEEE J. Sel. Top. Quantum Electron. 2, 847-860. 